\section{Efecto de las variantes de VKORC1 y CYP2C9 sobre la dosis de anticoagulantes orales en individuos chilenos}

\author{
FELIPE BENAVIDES ${ }^{1,2, a,{ }^{*},}$ NICOLE GROSSMAN ${ }^{1, \mathrm{~b}, *}$, HELENA POGGI ${ }^{2, \mathrm{a}}$, \\ ELENA NIETO ${ }^{2}$, ANTONIO BERTRÁN $^{3}$, DANIEL ARAOS $^{3}$, \\ MARCOS VÁSQUEZ ${ }^{1, \mathrm{a}}$, IGNAZ IBARRA ${ }^{1, \mathrm{~b}}$, FELIPE CÁCERES $^{1, \mathrm{~b}}$, \\ KARENA ESPINOZA ${ }^{1, \mathrm{c}}$, MARCELA LAGOS ${ }^{2}$, GABRIELA REPETTO M. ${ }^{1}$
}

\section{Effect of VKORC1 and CYP2C9 variants on dosage of oral anticoagulants in Chilean individuals}

Background: The dose of oral anticoagulants $(O A C)$ shows great variability among patients. Pharmacogenetic studies have shown that common variants in genes CYP2C9 ( ${ }^{\star} 2$ and $\left.{ }^{\star} 3\right)$ and VKORC1 $(-1639 G>A)$ are associated with lower requirements of OAC. Aim: To study the association between average maintenance doses of oral anticoagulant therapy required to maintain a stable INR and CYP2C9 and VKORC1 gene variants in Chilean adults. Material and Methods: Prospective study of patients on anticoagulant treatment and with a stable international normalized ratio (INR) for prothrombin time for at least three months. Patients were classified as having high or low acenocoumarol or warfarin requirements. Peripheral blood DNA genotyping was performed by polymerase chain reaction and restriction fragment polymorphism or sequencing and electrophoresis. Results: The study included 185 patients, 125 on acenocoumarol and 60 on warfarin. Patients with VKORC1-1639A allele were more likely to require lower doses of both drugs than patients with the $G$ allele (Odds ratio [OR] for acenocoumarol 9.06, and OR for warfarin $=18.7$ ). There was no association between CYP2C9 2 and ${ }^{\star} 3$ and acenocoumarol or warfarin requirements. Conclusions: There is an association between VKORC1-1639A variant and anticoagulant doses.

(Rev Med Chile 2015; 143: 1369-1376)

Key words: Acenocoumarol; Cytochrome P-450 CYP2C;, Pharmacogenetics; VKORC1, protein, human; Warfarin.
${ }^{1}$ Centro de Genética y Genómica, Facultad de Medicina, Clínica Alemana-Universidad del Desarrollo, Santiago, Chile. ${ }^{2}$ Departamento de Laboratorios Clínicos, Laboratorio de Biología Molecular y Citogenética, Facultad de Medicina, Pontificia Universidad Católica de Chile, Santiago, Chile.

3Laboratorio Clínico, Clínica Alemana de Santiago, Chile.

aBioquímico.

bEstudiante de Medicina, Facultad de Medicina, Clínica AlemanaUniversidad del Desarrollo. 'Tecnólogo Médico.

*Ambos autores contribuyeron de manera similar a este trabajo.

Financiado por Departamento Científico Docente Clínica Alemana de Santiago y el Departamento de Laboratorios Clínicos de la Facultad de Medicina Pontificia Universidad Católica de Chile.

Recibido el 31 de octubre de 2014, aceptado el 6 de agosto de 2015.

\section{Correspondencia a:}

Dra. Gabriela Repetto Av. Las Condes 12438, Lo Barnechea, Santiago, Chile. grepetto@udd.cl
L

os anticoagulantes orales (ACO) son utilizados en la prevención primaria y secundaria de patologías trombóticas y embólicas. En Chile, se utiliza tanto acenocumarol como warfarina, dependiendo de la preferencia del centro médico ${ }^{1}$. Actualmente, la prescripción de los ACO se realiza en base a la determinación del INR, ajustado a sexo y edad, controlando semanal y luego mensualmente hasta alcanzar los niveles requeridos. En el caso de ambos anticoagulantes existe una alta variabilidad en la respuesta terapéutica $y$, en consecuencia, en la dosis requerida por cada paciente. Dado que los ACO tienen un estrecho intervalo terapéutico y que los efectos adversos por dosis altas o bajas son graves, es necesario definir rápidamente la dosis exacta para cada paciente ${ }^{2,3}$. 
El efecto de los ACOs se mide utilizando el tiempo de protrombina (TP) y se expresa como International Normalized Ratio (INR). El INR se utiliza para estandarizar los resultados entre distintas preparaciones de tromboplastina usadas para medir el $\mathrm{TP}^{4}$. La dosis de inicio requerida en adultos para lograr un INR entre 2 y 3 varía ampliamente $(2 \text { a } 10 \mathrm{mg} \text { de warfarina por día })^{3}$. La variabilidad en los requerimientos depende de factores como edad, superficie corporal, función hepática e interacciones medicamentosas. Además, factores genéticos tienen un rol importante en la variabilidad inter-individual ${ }^{5}$.

Los ACO son antagonistas de la enzima vitamina K-epóxido-reductasa (codificada por el gen $V K O R C 1$ ), que tiene como función transformar la vitamina $\mathrm{K}(\mathrm{VK})$ de su estado epóxido a VK redu$\mathrm{cida}^{5}$. La VK es cofactor de la enzima $\gamma$-carboxilasa, que participa en la formación de los factores de la coagulación II, VII, IX y X $\mathrm{X}^{5}$. El catabolismo de los ACO es mediado por la enzima CYP2C9, que cataliza la conversión a metabolitos inactivos de la (S)-warfarina (la forma más activa) y de ambos enantiómeros (R/S) del acenocumarol ${ }^{6,7}$.

Estudios en distintas poblaciones revelan la presencia de variantes genéticas o polimorfismos que modifican las dosis requeridas de ACO. Se trata de las variantes $C Y P 2 C 9^{\star} 2$ y 3 y VKORC1 $-1639 \mathrm{G}>\mathrm{A}$, que se asocian a mayor sensibilidad frente a ACOs, menores requerimientos de dosis y mayor riesgo de sangrado. Esto ha dado origen a algoritmos que incluyen información clínica y genética para predecir las dosis de inicio y mantención, con buenos resultados, particularmente en pacientes que requieren dosis extremas ${ }^{3,8,9}$. Estas variantes han sido evaluadas en poblaciones caucásicas, asiáticas y africanas, existiendo diferencias en las frecuencias alélicas entre estos grupos. A la fecha, no existen publicaciones de análisis de polimorfismos en estos genes en población chilena.

En este estudio, se determinó las frecuencias alélicas y genotípicas de las variantes $C Y P 2 C 9^{\star} 2$ y *3 y VKORC1 -1639G >A, y se evaluó su asociación con la dosis de ACO requerida para mantener un INR estable ( 2 a 3 ) en pacientes chilenos.

\section{Pacientes y Métodos}

Se enrolaron, previo consentimiento informado, a adultos en tratamiento anticoagulante oral (TACO) de Clínica Alemana de Santiago (CAS) y del Programa de Control de TACO del Centro Médico San Joaquín (CMSJ) de la Red de Salud UC, recibiendo acenocumarol (Neo-Sintrom ${ }^{\circledR}$ ) o warfarina (Coumadin ${ }^{\circledR}$ ), que llevaban al menos 3 meses con INR estable. Los pacientes fueron clasificados en dos grupos: aquellos con requerimiento de dosis bajas (acenocumarol $\leq 7 \mathrm{mg}$ / semana o warfarina $\leq 22 \mathrm{mg} /$ semana) y aquellos con requerimientos mayores a esas dosis (dosis habitual). Se registró edad, sexo, peso, talla, dosis requerida y administración de otros fármacos. El estudio fue aprobado por el Comité de Ética de cada institución, de acuerdo a los términos de la declaración de Helsinki ${ }^{10}$.

\section{Genotipificación}

Se obtuvo muestras de sangre periférica y se extrajo ADN por columnas de silicio y por el método de Lahiri ${ }^{11}$. La genotipificación de los pacientes de CAS se realizó a través de reacción en cadena de la polimerasa (PCR), seguida de digestión mediante enzimas de restricción (PCR-RFLP), descrita previamente ${ }^{12,13}$. Para validar esta metodología se realizó secuenciación de estos fragmentos en pacientes heterocigotos y homocigotos para cada variante. El genotipo de los pacientes del CMSJ se estudió por secuenciación y posterior análisis por electroforesis capilar (ABI PRISM 3130, Applied Biosystems, Carslbad, CA, EE.UU.) usando partidores descritos previamente, según protocolo del fabricante ${ }^{12,13}$.

\section{Análisis estadístico}

Los promedios de dosis para cada genotipo se compararon mediante la prueba de Kruskal-Wallis, con test a posteriori de Dunn. Se utilizó media y desviación estándar para el análisis de la edad y dosis requerida. Para cada fármaco, se estableció la relación entre dosis y polimorfismos mediante modelo de regresión logística con SNPStat ${ }^{14}$. Se determinó la frecuencia alélica y genotípica de cada variante $y$, posteriormente, se realizó una prueba $\chi^{2}$ de Pearson para comprobar el equilibrio de Hardy-Weinberg. Para estudiar la magnitud de asociación entre variantes y la dosis baja, se calculó una razón de probabilidades (Odds Ratio, OR), evaluando diferentes modelos de herencia para cada gen y utilizando como criterio de selección el Bayesian Information Criterion ${ }^{15}$. Los análisis estadísticos fueron realizados con el programa GraphPad Prism 5.0, GraphPad Software (La Jolla, CA, EE.UU.). 


\section{Resultados}

Se enrolaron 185 pacientes, 101 de CAS y 84 de CMSJ. Se excluyeron 14 por datos incompletos, continuando en el estudio 170 pacientes, de los cuales 96 eran de CAS y 74 de CMSJ. La descripción demográfica está en la Tabla 1.

\section{Efecto de VKORC1 -1639G $>$ A en los}

requerimientos de $A C O$

Las frecuencias alélicas y genotípicas del polimorfismo VKORC1 -1639G $>$ A se describen en la Tabla 2. No se encontraron desviaciones del equilibrio de Hardy-Weinberg en ninguna de las variantes estudiadas (Tabla 2). El número de pacientes que requerían dosis bajas y habituales para cada genotipo de VKORC1 se muestran en la Tabla 3. Al agrupar los pacientes por genotipo, el promedio de dosis de acenocumarol fue de 19,4 (GG), 12,5 (GA) y $8,2 \mathrm{mg} / \mathrm{semana}$ (AA). Para warfarina, las dosis promedio fueron $36,0,31,8 \mathrm{y}$ $16,9 \mathrm{mg} / \mathrm{semana}$ (genotipos GG, GA y AA). Las diferencias entre los genotipos fueron estadísticamente significativas tanto para acenocumarol $(\mathrm{K}=43,83 ; \mathrm{p}<0,0001)$, como para warfarina $(\mathrm{K}=13,30 ; \mathrm{p}=0,0013)$. El test de Dunn a posteriori mostró diferencias significativas entre todos los grupos para acenocumarol mientras que en el caso de warfarina, la diferencia entre las dosis requeridas por los pacientes con genotipos $\mathrm{G} / \mathrm{G}$ y A/A no fue significativa (Figura 1). Utilizando un modelo de regresión logística se estudió la

Tabla 1. Caracterización demográfica de la población

\begin{tabular}{|c|c|c|}
\hline & Acenocumarol & Warfarina \\
\hline n Pacientes, (CAS/CMSJ) & $112(37 / 75)$ & $58 \quad(58 / 0)$ \\
\hline Mujeres (\%) & $59 \quad(52,7 \%)$ & $22 \quad(40 \%)$ \\
\hline $\bar{x}$ Edad (rango) & $67 \quad(19-89)$ & $62 \quad(25-86)$ \\
\hline$\overline{\mathrm{x}} \mathrm{IMC}$ (rango) & $27 \quad(20-42)$ & $27 \quad(21-38)$ \\
\hline$\overline{\mathrm{x}}$ Dosis $^{\ddagger}$ (rango) mg/semana & $13,2(3-56)$ & $29,4(4-75)$ \\
\hline
\end{tabular}

Tabla 2. Frecuencia alélica y genotípica de las variantes estudiadas

\begin{tabular}{|c|c|c|c|c|c|c|c|c|c|}
\hline \multirow{4}{*}{$\begin{array}{l}\text { VKORC1 } \\
-1639\end{array}$} & \multicolumn{6}{|c|}{ Frecuencia genotípica observada } & & & \\
\hline & \multirow{2}{*}{\multicolumn{2}{|c|}{$\begin{array}{c}\text { G/G } \\
43 \\
(25,3 \%)\end{array}$}} & \multirow{2}{*}{$\begin{array}{c}\text { G/A } \\
84 \\
(49,4 \%)\end{array}$} & \multicolumn{3}{|c|}{$\mathrm{A} / \mathrm{A}$} & \multirow{2}{*}{\multicolumn{3}{|c|}{$\begin{array}{c}\text { Frecuencia alélica } \\
\text { observada }\end{array}$}} \\
\hline & & & & \multicolumn{3}{|c|}{$\begin{array}{c}43 \\
(25,3 \%)\end{array}$} & & & \\
\hline & \multicolumn{6}{|c|}{ Frecuencia genotípica esperada } & G & & A \\
\hline & \multicolumn{2}{|c|}{$\begin{array}{c}42,5 \\
(25 \%)\end{array}$} & $\begin{array}{c}85 \\
(50 \%)\end{array}$ & \multicolumn{3}{|c|}{$\begin{array}{c}42,5 \\
(25 \%)\end{array}$} & 0,50 & & 0,50 \\
\hline \multirow[t]{2}{*}{ Valor $p$} & \multicolumn{6}{|c|}{$0,878^{*}$} & \multirow{3}{*}{\multicolumn{3}{|c|}{$\begin{array}{c}\text { Frecuencia alélica } \\
\text { observada }\end{array}$}} \\
\hline & \multicolumn{6}{|c|}{ Frecuencia genotípica observada } & & & \\
\hline \multirow{4}{*}{ CYP2C9 } & $* 1 / * 1$ & $* 1 / * 2$ & *1/*3 & $* 2 / * 2$ & $* 2 / * 3$ & $* 3 / * 3$ & & & \\
\hline & $\begin{array}{c}120 \\
(70,6 \%)\end{array}$ & $\begin{array}{c}29 \\
(17,1 \%)\end{array}$ & $\begin{array}{c}13 \\
(7,65 \%)\end{array}$ & $\begin{array}{c}2 \\
(1,17 \%)\end{array}$ & $\begin{array}{c}5 \\
(2,94 \%)\end{array}$ & $\begin{array}{c}1 \\
(0,58 \%)\end{array}$ & & & \\
\hline & \multicolumn{6}{|c|}{ Frecuencia genotípica esperada } & $* 1$ & *2 & *3 \\
\hline & $\begin{array}{c}116,95 \\
(68.79 \%)\end{array}$ & $\begin{array}{c}31,52 \\
(18,54 \%)\end{array}$ & $\begin{array}{c}16,59 \\
(9,76 \%)\end{array}$ & $\begin{array}{c}2,12 \\
(1,25 \%)\end{array}$ & $\begin{array}{c}2,24 \\
(1,31 \%)\end{array}$ & $\begin{array}{c}0,59 \\
(0,35 \%)\end{array}$ & 0,829 & 0,112 & 0,059 \\
\hline Valor $\mathrm{p}$ & \multicolumn{9}{|c|}{$0,189 *$} \\
\hline
\end{tabular}

*No significativo. 
Tabla 3. Dosis requerida para cada genotipo VKORC1

\begin{tabular}{|c|c|c|c|c|c|c|c|c|}
\hline \multirow{3}{*}{ Genotipo } & \multicolumn{4}{|c|}{ Acenocumarol } & \multicolumn{4}{|c|}{ Warfarina } \\
\hline & \multicolumn{2}{|c|}{$\begin{aligned} & \text { Dosis baja } \\
< & 7 \mathrm{mg} / \text { semana }\end{aligned}$} & \multicolumn{2}{|c|}{ Dosis habitual } & \multicolumn{2}{|c|}{$\begin{array}{c}\text { Dosis baja } \\
<22 \mathrm{mg} / \text { semana }\end{array}$} & \multicolumn{2}{|c|}{ Dosis habitual } \\
\hline & $\mathbf{n}$ & $\%$ & $\mathbf{n}$ & $\%$ & $\mathbf{n}$ & $\%$ & $\mathbf{n}$ & $\%$ \\
\hline-1639 GG & 0 & 0 & 30 & 31,6 & 3 & 25 & 10 & 21,7 \\
\hline$-1639 \mathrm{GA}$ & 5 & 29,4 & 47 & 49,5 & 1 & 8,3 & 31 & 67,4 \\
\hline-1639 AA & 12 & 70,6 & 18 & 18,9 & 8 & 66,6 & 5 & 10,9 \\
\hline Total & 17 & 100 & 95 & 100 & 12 & 100 & 46 & 100 \\
\hline
\end{tabular}

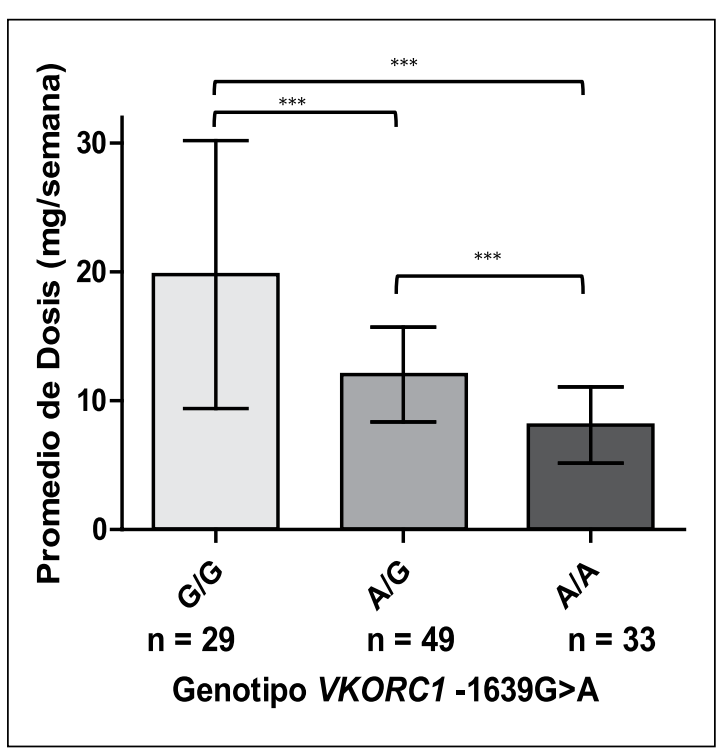

Figura 1. Genotipo de VKORC1 $-1639 \mathrm{G}>\mathrm{A}$ vs promedio de dosis de acenocumarol; $p<0,0001$ Kruskal-Wallis $(K=43,83)$.Test a posteriori de Dunn; ${ }^{* * *} p<0,001$; ${ }^{* *} p<0,01$. Barras indican Desviación Estándar (DE).

asociación entre el tipo de dosis y la presencia del alelo A de VKORC1 -1639, los resultados se muestran en la Tabla 6.

\section{Efecto de CYP2C9 en los requerimientos de anticoagulantes orales}

Las frecuencias alélicas y genotípicas obtenidas para las variantes ${ }^{\star} 2$ y ${ }^{\star} 3$ (variantes, var) y la variante nativa $\left({ }^{*} 1\right.$ o wild type, $\left.w t\right)$ del gen CYP2C9 se muestran en la Tabla 2. El número de pacientes que requieren dosis bajas y habituales

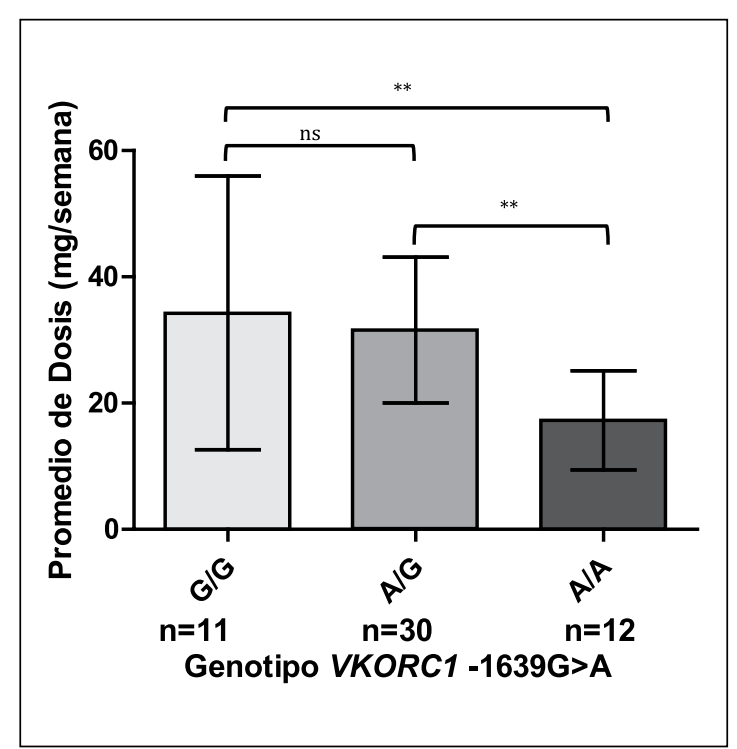

Figura 2. Genotipo de VKORC1 -1639G > A vs promedio de dosis de warfarina; $p=0,0013$ Kruskal-Wallis $(K=13,30)$. Test a posteriori de Dunn; ${ }^{* *} \mathrm{p}<0,001 ;{ }^{* *} \mathrm{p}<0,01$; ns $=$ no significativo. Barras indican Desviación Estándar (DE).

para cada genotipo de CYP2C9 se muestran en la Tablas 4 y 5. Los promedios de las dosis por genotipos agrupados ( $w t / w t, w t / v a r$ y var/var) tanto de acenocumarol $(13,5,13,4$ y $9,1 \mathrm{mg} / \mathrm{se}-$ mana) y warfarina $(31,2,25,7$ y $26,3 \mathrm{mg} /$ semana $)$ no mostraron diferencias significativas $(\mathrm{K}=1,46$; $p=0,48$ y $K=1,05 ; p=0,59$, respectivamente). En el modelo de regresión logística no se encontró ninguna asociación entre dosis baja y la presencia de la variante $C Y P 2 C 9^{\star} 2 \mathrm{o}^{*} 3$ tanto para acenocumarol como para warfarina (Tabla 6). 
Tabla 4. Dosis requerida para cada genotipo CYP2C9

\begin{tabular}{|c|c|c|c|c|c|c|c|c|}
\hline \multirow{3}{*}{ Genotipo } & \multicolumn{4}{|c|}{ Acenocumarol } & \multicolumn{4}{|c|}{ Warfarina } \\
\hline & \multicolumn{2}{|c|}{$\begin{aligned} & \text { Dosis baja } \\
< & 7 \mathrm{mg} / \text { semana }\end{aligned}$} & \multicolumn{2}{|c|}{ Dosis habitual } & \multicolumn{2}{|c|}{$\begin{array}{c}\text { Dosis baja } \\
<22 \mathrm{mg} / \mathrm{semana}\end{array}$} & \multicolumn{2}{|c|}{$\begin{array}{c}\text { Dosis habitual } \\
\text { Warfarina }\end{array}$} \\
\hline & $\mathbf{n}$ & $\%$ & $\mathbf{n}$ & $\%$ & $\mathbf{n}$ & $\%$ & $\mathbf{n}$ & $\%$ \\
\hline${ }^{*} 1 /{ }^{*} 1$ & 11 & 64,7 & 70 & 73,7 & 5 & 41,7 & 34 & 73,9 \\
\hline$* 1 / * 2$ & 2 & 11,8 & 15 & 15,8 & 4 & 33,3 & 8 & 17,4 \\
\hline$* 1 / * 3$ & 2 & 11,8 & 5 & 5,3 & 3 & 25,0 & 3 & 6,5 \\
\hline *2/*2 & 0 & 0 & 1 & 1,1 & 0 & 0 & 1 & 2,2 \\
\hline *2/*3 & 2 & 11,8 & 3 & 3,2 & 0 & 0 & 0 & 0 \\
\hline *3/*3 & 0 & 0 & 1 & 1,1 & 0 & 0 & 0 & 0 \\
\hline Total & 17 & 100 & 95 & 100 & 12 & 100 & 46 & 100 \\
\hline
\end{tabular}

Tabla 5. Dosis requerida para cada genotipo CYP2C9, agrupando variantes $2 *$ y 3

\begin{tabular}{|c|c|c|c|c|c|c|c|c|}
\hline \multirow{3}{*}{ Genotipo } & \multicolumn{4}{|c|}{ Acenocumarol } & \multicolumn{4}{|c|}{ Warfarina } \\
\hline & \multicolumn{2}{|c|}{$\begin{array}{c}\text { Dosis Baja } \\
<7 \mathrm{mg} / \text { semana }\end{array}$} & \multicolumn{2}{|c|}{ Dosis Habitual } & \multicolumn{2}{|c|}{$\begin{array}{c}\text { Dosis Baja } \\
<22 \mathrm{mg} / \text { semana }\end{array}$} & \multicolumn{2}{|c|}{ Dosis Habitual } \\
\hline & $\mathbf{n}$ & $\%$ & $\mathbf{n}$ & $\%$ & $\mathbf{n}$ & $\%$ & $\mathbf{n}$ & $\%$ \\
\hline wt/wt & 11 & 64,7 & 70 & 73,7 & 5 & 41,7 & 34 & 73,9 \\
\hline wt/var & 4 & 23,5 & 20 & 21,1 & 7 & 58,3 & 11 & 23,9 \\
\hline var/var & 2 & 11,8 & 5 & 5,3 & 0 & 0,0 & 1 & 2,2 \\
\hline Total & 17 & 100 & 95 & 100 & 12 & 100 & 46 & 100 \\
\hline
\end{tabular}

Tabla 6. Análisis de asociación entre la dosis requerida y variantes de los genes VKORC1 y CYP2C9

\begin{tabular}{|lllcc|}
\hline Fármaco & & Variante & OR (95\% IC) & Valor p \\
Acenocumarol & $(\mathrm{n}=112)$ & VKORC1 $-1639 \mathrm{G}>\mathrm{A}$ & $9,60(2,87-32,12)$ & $<0,0001$ \\
& & CYP2C9*2 & $1,26(0,38-4,15)$ & 0,71 \\
& & CYP2C9*3 & $2,62(0,75-9,19)$ & 0,15 \\
Warfarina & $(\mathrm{n}=58)$ & VKORC1 $-1639 \mathrm{G}>\mathrm{A}$ & $18,71(2,75-127,12)$ & 0,0007 \\
& & CYP2C9*2 & $1,41(0,35-5,63)$ & 0,63 \\
& & CYP2C9*3 & $12,61(0,88-181,30)$ & 0,038 \\
\hline
\end{tabular}

\section{Discusión}

Los fármacos anticoagulantes tienen un estrecho índice terapéutico, por lo que es difícil estimar la dosis adecuada para mantener un INR en rango terapéutico ${ }^{3}$. La dosis requerida puede variar en forma importante entre los distintos pacientes, lo que se explica por distintos factores como: edad, sexo, hábitos alimentarios, fármacos concomitantes y comorbilidades, entre otros. Sin embargo, la mayor influencia sobre la dosis de
ACO es atribuible a factores genéticos (40\%), a diferencia de otras patologías multifactoriales donde estos factores sólo explican $10 \%$ de la variación entre individuos ${ }^{3,16}$. Diversos estudios han demostrado que de todos los polimorfismos encontrados, los más importantes en cuanto a su asociación con dosis son $-1639 \mathrm{G}>\mathrm{A}$ en el gen VKORC1 y CYP2C9* 2 y $3^{3}$.

En estudios en distintas poblaciones, los polimorfismos en los genes $V K O R C 1$ y CY2PC9 tienen mayor implicancia en pacientes con re- 
querimientos menores de ACO. Este es el primer estudio farmacogenético de estas variantes en pacientes chilenos en tratamiento con ACO. Las frecuencias alélicas y genotípicas observadas son propias de nuestra población, con algunas similitudes a poblaciones europeas caucásicas y asiáticas sugerentes de una mezcla poblacional ${ }^{17,18}$. Frecuencias alélicas y genotípicas de $C Y P 2 C 9^{\star} 2$ y *3 en población general son bastante similares a las observadas en nuestros pacientes ${ }^{19}$.

De todos los pacientes estudiados, 29 requirieron dosis bajas (17\%), lo cual se encuentra dentro del porcentaje esperado ${ }^{20}$.

Los resultados obtenidos sugieren una asociación entre el genotipo VKORC1 y la dosis, lo que queda en evidencia al agrupar los pacientes según la dosis requerida: la mayoría de los pacientes homocigotos GG requirieron dosis habituales y cerca de la mitad de los homocigotos AA requirieron dosis bajas.

El OR ajustado, obtenido por análisis de asociación entre dosis y genotipo, demuestra una fuerte asociación entre el alelo A en VKORC1 y bajos requerimientos de ambos fármacos.

El tamizaje genético podría ayudar a identificar a los pacientes que requieren dosis habituales $o$ que pueden requirir dosis baja.

Al comparar el promedio de dosis requerida de ambos ACOs según los genotipos de CYP2C9, no se encontraron diferencias significativas entre los grupos. Investigaciones previas en población española encontraron una fuerte asociación entre requerimiento de dosis bajas y el alelo ${ }^{\star} 3$, mientras que el alelo ${ }^{\star} 2$ presenta una asociación menor ${ }^{21}$. Es posible que la baja frecuencia de las variantes de CYP2C9 y el insuficiente número de pacientes no permitiera determinar esta asociación en este estudio. Sin embargo, en el caso del alelo ${ }^{\star} 3$, que también presenta una baja frecuencia en la población, se encontró una asociación con dosis baja de warfarina (OR: 12,61), lo que podría deberse a que el alelo ${ }^{\star} 3$ produce una disminución más pronunciada sobre la actividad enzimática de CYP2C9 menor a 5\% versus alrededor de $15 \%$ para el alelo ${ }^{\star} 2$, ambas en comparación con la variante $\mathrm{WT}^{7,22}$.

Las proteínas que participan en la cascada de coagulación tienen diferente vida media, por lo que, hasta la inhibición completa de estos factores, el paciente requiere monitoreo continuo y hospitalización, lo que se traduce en un alto costo, tanto para el paciente como para el sistema de salud ${ }^{23}$.

Hoy en día, se reconoce cada vez más la importancia del estudio farmacogenético para establecer la dosis en pacientes en tratamiento anticoagulante, lo que se ve reflejado en una modificación en el año 2007 del etiquetado de la warfarina por la FDA (U.S. Food and Drug Administration). En el folleto se indica que la dosis inicial de 2 a $5 \mathrm{mg}$ diarios debe ser reducida en pacientes en los que se conozca la presencia de variantes genéticas de las enzimas CYP2C9 y VKORC1. Asimismo, en los últimos años se han publicado algoritmos de dosis de mantenimiento ${ }^{24,25}$, que han permitido crear una página web que permite a los clínicos monitorizar y estimar la dosis inicial y de mantenimiento de sus pacientes en tratamiento con warfarina (www.warfarindosing.org). La American Heart Association reconoce la existencia de un efecto de VKORC1 y CYP2C9 en la dosis requerida, pero no hace recomendaciones al respecto ${ }^{26}$. Es así como la discusión sobre la relación costo-beneficio de estudiar estas variantes en los pacientes sigue vigente. En el estudio de Lindner y cols. ${ }^{27}$, se estableció un modelo de análisis de decisión para simular los resultados clínicos y económicos del tratamiento con acenocumarol con y sin tamizaje genético de los pacientes, dando como resultado un mayor costo monetario, pero menor número de episodios de hemorragia al realizar el análisis genético en los pacientes. Con respecto a la estabilización de la dosis mediante el uso de estas variantes, Kimmel et al. ${ }^{28}$ demostraron que no existe diferencia con la aproximación clínica en un período de 4 semanas. Sin embargo, es en la primera semana de tratamiento donde conocer el genotipo del paciente permitiría comenzar con la dosis adecuada, interrogante que sigue siendo motivo de estudio y discusión. En 2010, Epstein et al. determinaron que la genotipificación de warfarina reduce el riesgo de sangrado o tromboembolismo en pacientes que inician $\mathrm{ACO}^{16}$. A pesar de la gran utilidad que tienen los exámenes genéticos en Chile, aún tienen un costo elevado para la población, lo que dificulta su implementación práctica. Además, si bien la utilidad es reconocida, existe percepción de conocimiento insuficiente entre clínicos en Latinoamérica, lo que constituye una barrera para su uso ${ }^{29}$. Sin embargo, una vez que sean más asequibles y en el caso de pacientes donde no haya sido posible encontrar la dosis adecuada en las primeras $24 \mathrm{~h}$, la genotipificación sería de 
utilidad para reducir costos al paciente y a los centros de tratamiento, minimizar efectos adversos y aumentar la eficacia de los $\mathrm{ACO}^{30}$.

En conclusión, nuestro estudio demuestra que la dosis de mantenimiento tiene una fuerte asociación con la variante más frecuente en el gen VKORC1 (-1639G>A). No encontramos asociación con $C Y P 2 C 9$, lo que podría explicarse por su baja frecuencia en la población. Los resultados de este estudio representan la base para evaluar la posibilidad de introducir el tamizaje genético en pacientes chilenos con tratamiento anticoagulante, de forma de asistir a los clínicos en la predicción de la dosis requerida para reducir costos al paciente y centros de tratamiento, minimizar efectos adversos y aumentar la eficacia.

\section{Referencias}

1. Berkovits A, Aizman A, Zúñiga P, Pereira J, Mezzano D. Nuevos anticoagulantes orales. Rev Med Chile 2011; 139 (10): 1347-55.

2. Montes R, Nantes O, Molina E, Zozaya J, Hermida J. Predisposición genética al sangrado durante el tratamiento con anticoagulantes orales. An Sist Sanit Navar 2008; 31 (3): 247-57.

3. Rieder MJ, Reiner AP, Gage BF, Nickerson DA, Eby CS, McLeod HL, et al. Effect of VKORC1 haplotypes on transcriptional regulation and warfarin dose. $\mathrm{N}$ Engl J Med 2005; 352 (22): 2285-93.

4. Evans A, Davis S, Kilpatrick C, Gerraty R, Campbell D, Greenberg P. The morbidity related to atrial fibrillation at a tertiary centre in one year: $9 \%$ of all strokes are potentially preventable. J Clin Neurosci 2002; 9 (3): 268-72.

5. Schwarz UI, Ritchie MD, Bradford Y, Li C, Dudek SM, Frye-Anderson A, et al. Genetic determinants of response to warfarin during initial anticoagulation. $\mathrm{N}$ Engl J Med 2008; 358 (10): 999-1008.

6. Aithal GP, Day CP, Kesteven PJ, Daly AK. Association of polymorphisms in the cytochrome P450 CYP2C9 with warfarin dose requirements and risk of bleeding complications. Lancet 1999; 353 (9154): 717-9.

7. Xie H, Prasad HC, Kim RB, Stein CM. CYP2C9 allelic variants: ethnic distribution and functional significance. Adv Drug Deliv Rev 2002; 54 (10): 1257-70.

8. The International Warfarin Pharmacogenetics Consortium (Estimation of the warfarin dose with clinical and pharmacogenetic data. N Eng J Med 2009; 360 (8): 753-64.
9. Verde Z, Ruiz JR, Santiago C, Valle B, Bandrés F, Calvo $\mathrm{E}$, et al. Single algorithm approach to predict acenocoumarol dose based on CYP2C9 and VKORC1 allele variants. PLoS ONE 2010; 5 (6): e11210.

10. World Medical Association Inc. Declaration of Helsinki. Ethical principles for medical research involving human subjects. J Indian Med Assoc 2009; 107 (6): 403. Disponible en www.wma.net [Consultado el 13 de marzo de 2014]

11. Lahiri D, Nurnberger, J. A rapid non-enzymatic method for the preparation of HMW DNA from blood for RFL Pstudies. Nucleic Acids Res 1991; 19 (19): 5444.

12. Sconce E, Khan TI, Wynne HA, Avery P, Monkhouse L, King BP. The impact of CYP2C9 and VKORC1 genetic polymorphism and patient characteristics upon warfarin dose requirements: proposal for a new dosing regimen. Blood 2005; 106 (7): 2329-33.

13. Veenstra DL, Blough DK, Higashi MK, Farin FM, Srinouanprachan S, RiederMJ. CYP2C9 haplotype structure in European American warfarin patients and association with clinical outcomes. Clin Pharmacol Ther 2005; 77 (5): 353-64.

14. Solé X, Guinó E, Valls J, Iniesta R, Moreno V. SNPStats: a web tool for the analysis of association studies. Bioinformatics 2006; 22 (15): 1928-9.

15. Kass R, Raftery AE. Bayes Factor. J Am Stat Assoc 1995; 90 (430): 773-95.

16. Epstein RS, Moyer TP, Aubert RE, O Kane DJ, Xia F, Verbrugge RR. Warfarin genotyping reduces hospitalization rates: The MM-WES (Medco-Mayo Warfarin Effectiveness Study). J Am Coll Cardiol 2010; 55 (25): 2804-12.

17. Cruz-Coke R, Moreno R. Genetic epidemiology of single gene defects in Chile. J Med Genet 1994; 31 (9): 702-6.

18. Rothhammer F, Moraga M, Santoro CM, Arriaza BT. Origen de los Changos. Análisis de ADNmt antiguo sugiere descendencia de pescadores de la cultura Chinchorro (7.900-4.000 A.P.). Rev Med Chile 2010; 138 (2): 251-6.

19. Scibona P, Redal M, Garfi L, Arbelbide J, Argibay, Belloso W. Prevalence of CYP2C9 and VKORC1 alleles in the Argentine population and implications for prescribing dosages of anticoagulants. Genet Mol Res 2012; 11 (1): 70-6.

20. Montes R, Ruiz de Gaona E, Martínez-González MA, Alberca I, Hermida J. The c.-1639G > A polymorphism of the VKORC1 gene is a major determinant of the response to acenocoumarol in anticoagulated patients. $\mathrm{Br}$ J Haematol 2006; 133 (2): 183-7.

21. Hermida J, Zarza J, Alberca I, Montes R, López ML, Molina E. Differential effects of $2 \mathrm{C} 9^{*} 3$ and $2 \mathrm{C} 9^{\star} 2$ va- 
riants of cytochrome P-450CYP2C9 on sensitivity to acenocoumarol. Blood 2002; 99 (11): 4237-9.

22. Kirchheiner J, Brockmöller J. Clinical consequences of cytochrome P450 2C9 polymorphisms. Clin Pharmacol Ther 2005; 77 (1): 1-16.

23. Yang J, Chen Y, Li X, Wei X, Chen X, Zhang L, et al. Influence of CYP2C9 and VKORC1 genotypes on the risk of hemorrhagic complications in warfarin-treated patients: A systematic review. Int J Cardiol 2013; 168 (4): 4234-43.

24. Perini JA, Struchiner CJ, Silva-Assunção E, Santana IS, Rangel F, Ojopi EB, et al. Pharmacogenetics of warfarin: development of a dosing algorithm for Brazilian patients. Clin Pharmacol Ther 2008; 84 (6): 722-8.

25. Lenzini PA, Grice GR, Milligan PE, Gatchel SK, Deych E, Eby CS, et al. Optimal initial dose adjustment of warfarin in orthopedic patients. Ann Pharmacother 2007; 41 (11): 1798-804.

26. Hirsh J, Fuster V, Ansell J, Halperin JL; American Heart Association; American College of Cardiology Founda- tion. American Heart Association/American College of Cardiology Foundation guide to warfarin therapy. Circulation 2003; 107 (12): 1692-711.

27. Lindner L, Lara N, Tàssies D, Tejedor D, Jofre-Monseny L. Coste-efectividad de la identificación genética en pacientes en tratamiento con acenocumarol. Rev Esp Econ Salud 2009; 8 (2): 77-84.

28. Kimmel SE, French B, Geller NL; COAG Investigators. Genotype-guided dosing of vitamin K antagonists. N Engl J Med 2014; 370 (18): 1763-4.

29. Quiñones LA, Lavanderos M, Cayun JP, García-Martin E, Agundez JA, Cáceres D, et al. Perception of the usefulness of drug/gene pairs and barriers for pharmacogenomics in Latin America. Curr Drug Metab 2014; 15 (2): 202-8.

30. Bodin L, Verstuyft C, Tregouet DA, Robert A, Dubert L, Funck-Brentano, et al. Cytochrome P450 2C9 (CYP2C9) and vitamin $\mathrm{K}$ epoxide reductase (VKORC1) genotypes as determinants of acenocoumarol sensitivity. Blood 2005; 106(1): 135-40. 